\title{
The value of hybrid interstitial tandem and ring applicators for organ at risk dose reduction in small volume cervical cancer
}

\author{
Najeeb Crossley, MD, Camrin Tipton, BS, Teresa Meier, MD, Mickaela Sudhoff, DMP, Jordan Kharofa, MD \\ Department of Radiation Oncology, University of Cincinnati Medical Center, Cincinnati, United States
}

\begin{abstract}
Purpose: Magnetic resonance imaging (MRI)-guided adaptive brachytherapy is the standard of care for cervical cancer. Hybrid intracavitary/interstitial applicators for bulky tumor (high-risk clinical target volume [HR-CTV] $>30 \mathrm{cc}$ ) dose escalation is recommended in the EMBRACE II trial. The value of hybrid applicators for smaller HR-CTV $(<30 \mathrm{cc})$ in organ at risk (OAR) sparing is less certain.

Material and methods: Twenty-seven patients with FIGO stage I-IVA cervical cancer treated with definitive chemoradiation and MRI-based brachytherapy using conventional tandem and ring (TR) applicators were re-planned using virtual needles. They were then summed with the external beam dose to evaluate target coverage and OAR dose

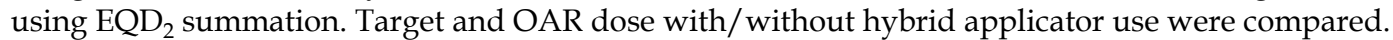

Results: Eighty-one percent had HR-CTV volumes $<30 \mathrm{cc}$, for which, hybrid TR applicators had significantly lower mean $\mathrm{D}_{2 \mathrm{cc}}$ to all OARs without differences in target coverage. For HR-CTV $<30 \mathrm{cc}$, the bladder and rectal OAR goals per EMBRACE II were exceeded in significantly fewer patients with the hybrid TR applicators. No significant difference was found in the sigmoid $\mathrm{D}_{2 \mathrm{cc}}$ dose goal.

Conclusions: In small volume tumors $(<30 \mathrm{cc})$, hybrid applicators may offer improved OAR sparing compared with conventional tandem and ring applicators, and may increase the proportion of patients meeting EMBRACE II OAR goals.

Key words: cervical cancer, brachytherapy, hybrid applicator.

\section{Purpose}

Brachytherapy is of critical importance in the treatment of locally advanced cervical cancer. Treatment is performed using a variety of applicators and imaging modalities. Use of magnetic resonance imaging (MRI)based planning in defining the target volume has become more widely available and is preferred to computed tomography (CT) due to improved soft tissue resolution. Accurate target delineation and dose limiting toxicities remain a challenge with CT-based approaches [1,2,3] A small prospective trial by Viswanathan et al. compared contours and dose volume histograms (DVH) of tumor and organs at risk (OAR) in cervical cancer brachytherapy. The MRI and CT DVH values for OAR were similar, but statistically significant differences were shown in the high-risk clinical target volume (HR-CTV) width when contoured on CT vs. MRI [4]. With improved target resolution, there has been increased interest in using customized hybrid intracavitary/interstitial applicators to customize dose for bulky tumors. Using MRI-based treat- ment planning techniques, hybrid applicators allow for an increase in target coverage, treated volume, and total dose without increasing the dose to critical structures [5]. The EMBRACE study has also provided a wealth of information regarding the clinical feasibility of hybrid intracavitary/interstitial applicators as well as improved DVH parameters and acceptable additional procedure time [6]. Based on the improvement in target dose and sparing of OAR found with the use of hybrid applicators in these studies, the GYN GEC-ESTRO dose constraints have been modified to support a higher HR-CTV $D_{90}$ value without a significant increase in dose to OAR [6]. Kirisits et al. showed that the use of a hybrid applicator for bulky tumors allows increases in target coverage, treated volume, and total dose without increasing the dose to critical structures [5]. The role of hybrid needle applicators in OAR dose reduction for small volume tumors is less clear. With increased understanding of potential toxicities with doses to organs at risk previously considered safe, the use of hybrid applicators in small
Address for correspondence: Najeeb Crossley, MD, Department of Radiation Oncology, University of Cincinnati Medical Center, 234 Goodman St., ML 0757, Cincinnati, OH, 45267, USA, ๑e-mail: crosslnb@ucmail.uc.edu
Received: 06.08 .2019

Accepted: 30.01 .2020

Published: 28.02.2020 
volume tumors may also be applied to improve the therapeutic ratio. The purpose of this study was to evaluate the benefit of hybrid tandem and ring applicators in OAR sparing for small volume tumors.

\section{Material and methods}

Twenty seven consecutive patients who underwent high-dose-rate (HDR) brachytherapy for cervical cancer between September 2015 and October 2017 at the University of Cincinnati were retrospectively reviewed. All patients were re-planned at fraction 1 with the use of virtual needles. Virtual needles were placed using a template for the tandem and Vienna ring cap (Mick Radio-Nuclear Instruments, Inc.) hybrid applicator. Needle positions were determined based on the tumor and OAR anatomy (Figure 1).
Needles were advanced to a maximum distance of $4 \mathrm{~cm}$ beyond the ring, and the first loading position was typically 2-3 mm back from the tip of the needle. Each fraction was then propagated to four total fractions (7 Gy $\times 4$ fractions) and summed with the external beam dose using $\mathrm{EQD}_{2}$ summation to evaluate differences in target coverage and organ at risk dose. Coverage of the HR-CTV and sparing of OARs was achieved by a graphical optimization method. All hybrid plans were compared to the actual plans treated without hybrid interstitial needles. A subset of small volume tumors $<30 \mathrm{cc}$ were compared. Tumors less than $30 \mathrm{cc}$ were defined as small or "limited" volume per the EMBRACE II protocol. The HR-CTV $D_{90}$ and $\mathrm{D}_{2 \mathrm{cc}}$ dose to bladder, rectum, and sigmoid were compared with a paired $t$-test.
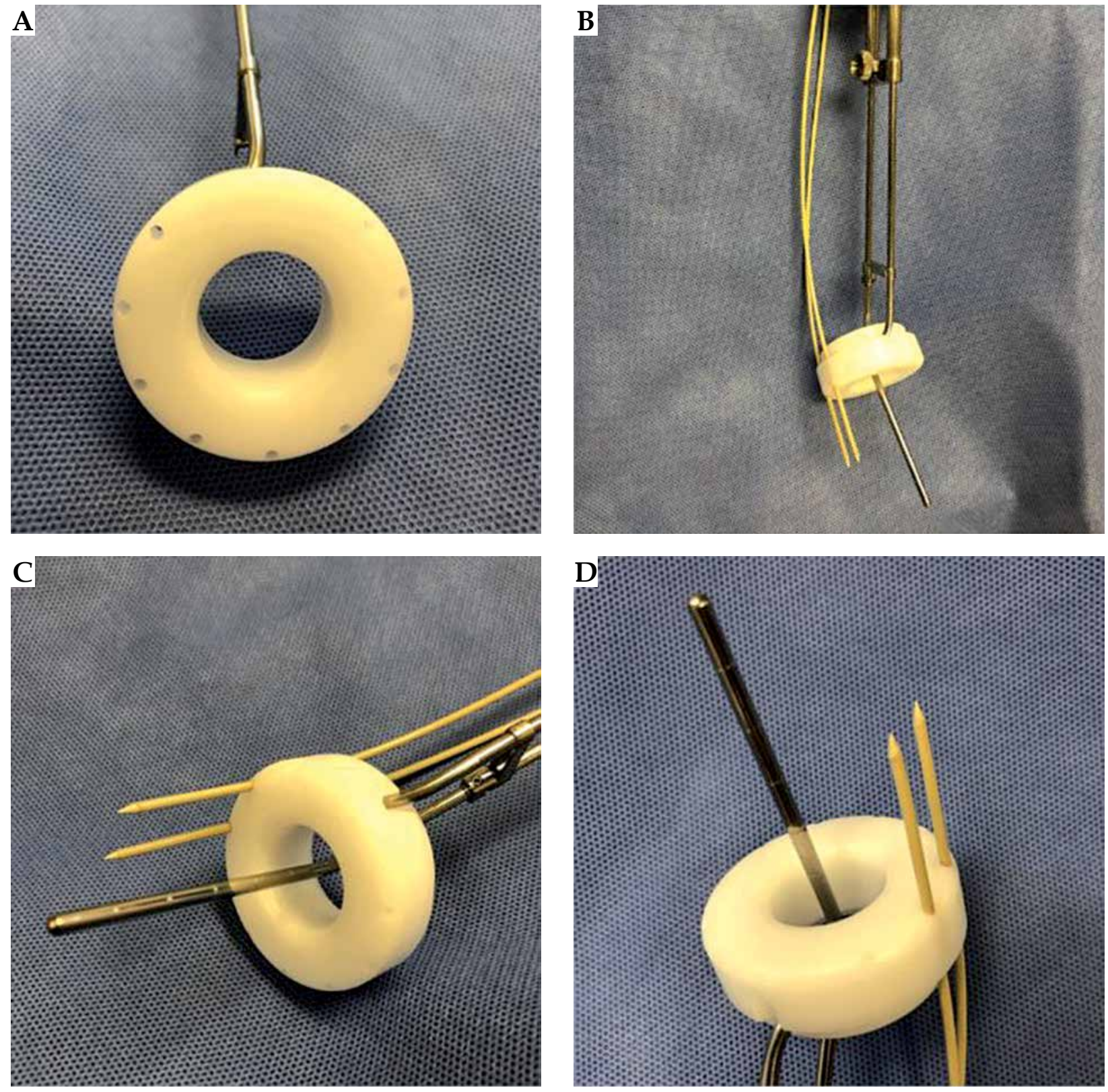

Fig. 1. Hybrid tandem and ring applicator 
A Fisher's exact test was performed to assess the proportion of patients achieving all the EMBRACE II constraints (bladder $\mathrm{D}_{2 \mathrm{cc}}<80 \mathrm{~Gy}$ as well as rectum and sigmoid < $65 \mathrm{~Gy}$ ) with and without the use of a hybrid applicator.

\section{Results}

Twenty-seven patients with International Federation of Gynecology and Obstetrics (FIGO) stage I-IVA cervical cancer were reviewed during the study period. The median age was 48 (range, 23-75). All patients were treated with definitive chemoradiation (45-50.4 Gy) and MRI-based brachytherapy using conventional tandem and ring applicators. Of the 27 patients, 24 (89\%) had MRI-based brachytherapy planning defined as at least one MRI performed with the applicator in place. Of the 27 patients, $8(30 \%)$ had FIGO stage I disease, $14(52 \%)$ had stage II, 4 (15\%) stage III, and 1 (3\%) had stage IVA disease. For all patients, the median HR-CTV volume was $24.4 \mathrm{cc}(16-55 \mathrm{cc})$. Twenty-two of the 27 patients $(81 \%)$ had small volume tumors, with HR-CTV volumes $<30 \mathrm{cc}$. Eighteen of those twenty-two patients had an MRI fused for brachytherapy planning.

Each of the 27 patients underwent re-planning with placement of virtual needles at fraction one. The median number of needles used was 2 (range, 1-4). At least 2 virtual needles were used in planning for the majority of patients ( 1 needle, $n=1(4 \%), 2$ needles, $n=12(44 \%)$, 3 needles, $n=7(26 \%)$, 4 needles, $n=7(22 \%))$.

For all patients, the hybrid tandem and ring (TR) applicators had a lower mean $\mathrm{D}_{2 \mathrm{cc}}$ to the bladder (71 Gy vs. $86 \mathrm{~Gy}, p<0.0001$ ), rectum (57 Gy vs. $62 \mathrm{~Gy}, p<0.0001$ ), and sigmoid (64 Gy vs. 67 Gy, $p=0.0039$ ) compared to conventional TR applicators, with no difference in HR-CTV D ${ }_{90}$ coverage (93.4 vs. $94.5, p=0.53$ ) (Table 1 ). In patients with HR-CTV $<30 \mathrm{cc}$, the hybrid TR applicators had a lower mean $D_{2 \mathrm{cc}}$ to the bladder (70 Gy vs. $85 \mathrm{~Gy}$, $p<0.0001$ ), rectum (57 Gy vs. $62 \mathrm{~Gy}, p<0.0001$ ), and sigmoid (64 Gy vs. $67 \mathrm{~Gy}, p=0.005$ ) compared to conventional TR applicators, without difference in HR-CTV $\mathrm{D}_{90}$ coverage (96 Gy vs. $94 \mathrm{~Gy}, p=0.12$ ) (Table 2). In patients with HR-CTV $<30 \mathrm{cc}$, the $\mathrm{D}_{2 \mathrm{cc}}$ bladder goal of $<80$ Gy as per EMBRACE II was exceeded in 1/22 (4.5\%) of patients, with the hybrid TR applicators compared to $17 / 22(77 \%)$ patients with conventional TR applicators $(p=0.0001)$. Similarly, more patients in the conventional TR group exceeded the $\mathrm{D}_{2 \mathrm{cc}}$ goal for rectum of $65 \mathrm{~Gy}$ $(7 / 22,32 \%)$ compared to the hybrid TR group $(0 / 22,0 \%)$ $(p<0.001)$. There was no difference in the proportion of patients with sigmoid $\mathrm{D}_{2 \mathrm{cc}}>65 \mathrm{~Gy}$ in the conventional TR group $(12 / 22,59 \%)$ compared to the hybrid TR group $(9 / 22,41 \%)(p=0.54)$. Figure 2 shows pre and post needle axial, coronal, and sagittal MRI images of the modification of dose.

\section{Discussion}

With the development of image-based adaptive brachytherapy for cervical cancer using MRI, uncertainties in target volumes and organs at risk have been reduced [7]. The ability to obtain essential information about tumor extent and response as well as to delineate structures utilizing adequate imaging protocols meets the demands for 3D image-based brachytherapy [8]. When analyzed systematically, MRI findings prior to external beam radiotherapy and at the time of brachytherapy provide this essential data [8,9]. Areas of high and intermediate intensity that represent macroscopic and partly microscopic residual disease must be included in the HR-CTV per GEC-ESTRO guidelines [10,11]. There have also been improvements in outcomes such as local control, overall survival, and morbidity with this MRI-based approach [12]. In 2008, the GEC-ESTRO GYN network initiated the EMBRACE study for the purpose of evaluating and benchmarking MRI-guided brachytherapy in a prospective multicenter fashion. Results from this study established a significant correlation between late rectal mor-

Table 1. HR-CTV coverage and OAR sparing for all patients (mean values in Gy), 95\% confidence intervals listed

\begin{tabular}{lccc} 
& Conventional & Hybrid & $p$-value \\
\hline HR-CTV $D_{90}$ & $94.5(\mathrm{Cl}$ 92.05-97.01) & $93.5(\mathrm{Cl}$ 91.75-95.42) & 0.53 \\
\hline Bladder $\mathrm{D}_{2 c c}$ & $85.8(\mathrm{Cl}$ 82.34-89.16) & $71.5(\mathrm{Cl}$ 68.73-74.18) & $<0.0001$ \\
\hline Rectum $\mathrm{D}_{2 c c}$ & $62.1(\mathrm{Cl}$ 60.41-63.87) & $57.4(\mathrm{Cl}$ 55.86-58.91) & $<0.0001$ \\
\hline Sigmoid $\mathrm{D}_{2 c c}$ & $66.6(\mathrm{Cl}$ 64.34-68.82) & $63.9(\mathrm{Cl}$ 61.69-66.28) & 0.0039
\end{tabular}

Table 2. HR-CTV < 30 cc OAR results (mean values in Gy), 95\% confidence intervals listed

\begin{tabular}{lllc} 
& Conventional & Hybrid & $p$-value \\
\hline HR-CTV $D_{90}(<30 c c)$ & $96.0(\mathrm{Cl}$ 93.49-98.58) & $93.6(\mathrm{Cl}$ 91.65-95.61) & 0.12 \\
\hline Bladder $\mathrm{D}_{2 c c}$ & $85.8(\mathrm{Cl} 82.26-89.41)$ & $70.7(\mathrm{Cl}$ 68.21-73.14) & $<0.0001$ \\
\hline Rectum $\mathrm{D}_{2 c c}$ & $62.4(\mathrm{Cl}$ 60.28-64.42) & $57.6(\mathrm{Cl}$ 55.75-59.39) & $<0.0001$ \\
\hline Sigmoid $\mathrm{D}_{2 c c}$ & $66.6(\mathrm{Cl}$ 64.04-69.09) & $63.9(\mathrm{Cl}$ 61.15-66.56) & 0.0039
\end{tabular}




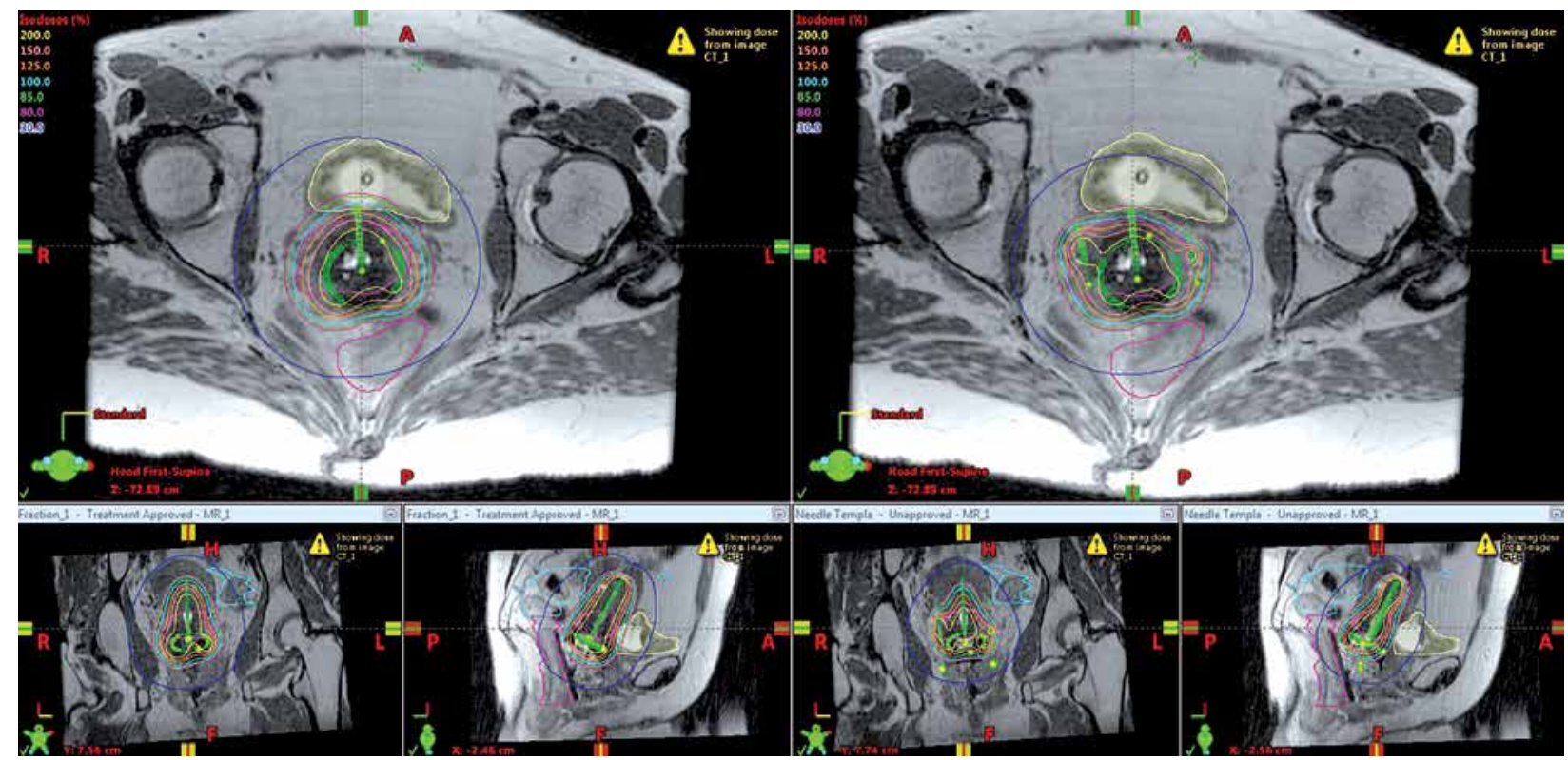

Fig. 2. Comparison of dose without and with needles in a 44-year-old patient with FIGO stage IIB cervical cancer with a HR-CTV volume of 19.5 cc. Pre and post needle values were as follows: HR-CTV D ${ }_{90} 102.1$ Gy vs. 101.7 Gy, bladder D 2 cc 85.4 Gy vs. 70.2 Gy, rectum 65.6 Gy vs. $61.4 \mathrm{~Gy}$, sigmoid $66.1 \mathrm{~Gy}$ vs. $60.6 \mathrm{~Gy}$

bidity and dose volume parameters. They found a $D_{2 c c}$ $\leq 65$ Gy to be associated with more minor and less frequent rectal morbidity, whereas a $\mathrm{D}_{2 \mathrm{cc}} \geq 75$ Gy was associated with more major and more frequent rectal morbidity [13]. When considering bladder toxicity, a significant dose relationship was also found, which indicated that at dose levels beyond $80 \mathrm{~Gy} \mathrm{EQD}_{2}$, there was a clinically significant increase in grade 2 or greater morbidity [14]. Similarly, Georg et al. showed that well defined dose response curves could be established for $\mathrm{D}_{2 \mathrm{cc}}$ in the rectum and urinary bladder. For rectal morbidity, they found a $5 \%$ incidence of $G 2$ to $G 4$ toxicity with a $D_{2 c c}$ dose of $67 \mathrm{~Gy}$. There was a 5\% incidence of G2 to G4 bladder complications with 70 Gy [15].

In addition, there is promising data regarding the use of hybrid applicators based on a retrospective analysis of 610 patients from the RetroEMBRACE study. This cohort of patients with locally advanced cervical cancer was treated with IGABT based on MRI or CT. The study involved 2 groups, one in which hybrid applicators were used systematically in more than $20 \%$ of patients and an intracavitary group, which included patients who were treated before systemic use of hybrid applicators was implemented [16]. Approximately, three quarters of the patients had locally advanced disease and $82 \%$ received IGABT based on MRI, and combined IC/IS (intracavitary/interstitial) brachytherapy was used in total $24 \%$ of patients. Overall, 3-year and 5-year local control rates were $92 \%$ and $89 \%$, respectively, and in the combined IC/IS group, 3-year and 5-year local control rates were $94 \%$ and $91 \%$, respectively. When comparing the IC and the IC/IS groups, local control was $5 \%$ better in the IC/IS group. Similarly, when looking at two groups with HR-CTV volume $\geq 30 \mathrm{cc}$ and $<30 \mathrm{cc}$, local control was again found to be superior in the IC/IS group at $92 \%$ and $87 \%$ at 3 and 5 years for large tumors. By comparison, the IC only group had $82 \%$ and $80 \%$ local control rates for large tumors. This difference resulted in a significant 3-year and 5-year survival difference of $10 \%$ and $7 \%$ between the groups. For small tumors $(<30 \mathrm{cc})$, local control in the IC/IS group was $97 \%$ at 3 and 5 years compared to $96 \%$ and $93 \%$ in the IC group. In contrast to that of large tumors, there was no significant difference found [16].

Based on this data, there is now sufficient evidence for the use of hybrid intracavitary/interstitial applicators for tumor control for large volume tumors. The EMBRACE II protocol has decreased the desired OAR goals for bladder and rectum in hope of decreasing the morbidity of therapy. The Bladder $\mathrm{D}_{2 \mathrm{cc}}$ goal was decreased to $80 \mathrm{~Gy}$ compared to 90 Gy in the EMBRACE I with hope of lowering grade 2 or higher urinary toxicity to $21 \%$ and grade 3 or higher urinary toxicity to $6 \%$. The rectal $\mathrm{D}_{2 \text { cc }}$ goal was decreased to < 65 Gy in the EMBRACE II compared to 70 Gy in the EMBRACE I protocol, with the goal of lowering grade 2 or higher rectal toxicity to $9 \%$ and grade 3 or higher rectal toxicity to $2 \%$. Mazeron et al. have reported, based on the EMBRACE cohort, that a $\mathrm{D}_{2 \mathrm{cc}}$ rectal dose greater than or equal to $75 \mathrm{~Gy}$ was associated with a $12.5 \%$ risk of fistula at 3 years compared to $0-2.7 \%$ at lower doses. A $D_{2 c c}$ dose of less than 65 Gy was associated with a twofold lower risk of proctitis than $65 \mathrm{~Gy}$ or greater [13].

These new GYN GEC ESTRO OAR parameters may be difficult to meet with conventional TR methods. This is evidenced by the results of our study in small volume tumors showing the $\mathrm{D}_{2 \mathrm{cc}}$ bladder goal of $<80$ Gy was exceeded in $4.5 \%$ of patients with the hybrid TR applicators vs. $77 \%$ of patients with conventional TR applicators. Additionally, more patients in our conventional TR group exceeded the $\mathrm{D}_{2 \mathrm{cc}}$ goal for rectum of $65 \mathrm{~Gy}$ at $32 \%$ compared to the hybrid TR group at $0 \%$.

Potential areas of weakness in our study include the small sample size from a single institution and the ret- 
rospective nature of the study. In addition, the generated hybrid plans were idealized virtual plans. The needle placement in virtual plans may be more difficult to achieve in practice. Lastly, vaginal doses were not analyzed in our study.

\section{Conclusions}

Hybrid intracavitary/interstitial applicators are an invaluable tool for enhancing HR-CTV coverage and escalating dose in large volume cervical cancers without an increase in the dose to critical structures.

The results of our study support the use of hybrid applicators in patients with small volume HR-CTVs and suggest that the EMBRACE II constraints will be met in a greater proportion of patients. Whether these potential dosimetric advantages will result in lower rates toxicity rates will need to be evaluated clinically.

\section{Acknowledgements}

The authors report no proprietary or commercial interest in any product mentioned or concept discussed in this article. This research did not receive any specific grant from funding agencies in the public, commercial, or not-for-profit sectors.

\section{Disclosure}

The authors report no conflict of interest.

\section{References}

1. Hopkins MP, Morley GW. Prognostic factors in advanced stage squamous cell cancer of the cervix. Cancer 1993; 72: 2389-2393.

2. Perez CA, Grigsby PW, Nene SM et al. Effect of tumor size on the prognosis of carcinoma of the uterine cervix treated with irradiation alone. Cancer 1992; 69: 2796-2806.

3. Pötter R, Knocke TH, Fellner C et al. Definitive radiotherapy based on HDR brachytherapy with iridium 192 in uterine cervix carcinoma: report on the Vienna University Hospital findings (1993-1997) compared to the preceding period in the context of ICRU 38 recommendations. Cancer Radiother 2000; 4: 159-172.

4. Viswanathan AN, Dimopoulos J, Kirisits C et al. Computed tomography versus magnetic resonance imaging-based contouring in cervical cancer brachytherapy: results of a prospective trial and preliminary guidelines for standardized contours. Int J Radiat Oncol Biol Phys 2007; 68: 491-498.

5. Kirisits C, Lang S, Dimopoulos J et al. The Vienna applicator for combined intracavitary and interstitial brachytherapy of cervical cancer: Design, application, treatment planning, and dosimetric results. Int J Radiat Oncol Biol Phys 2006; 65: 624630.

6. Fokdal L, Tanderup K, Hokland SB et al. Clinical feasibility of combined intracavitary/interstitial brachytherapy in locally advanced cervical cancer employing MRI with a tan$\mathrm{dem} /$ ring applicator in situ and virtual preplanning of the interstitial component. Radiother Oncol 2013; 107: 63-68.

7. Dimopoulos JCA, Petrow P, Tanderup K et al. Recommendations from Gynaecological (GYN) GEC-ESTRO Working Group (IV): Basic principles and parameters for MR imaging within the frame of image based adaptive cervix cancer brachytherapy. Radiother Oncol 2012; 103: 113-122.
8. Dimopoulos JCA, Schard G, Berger D et al. Systematic evaluation of MRI findings in different stages of treatment of cervical cancer: Potential of MRI on delineation of target, pathoanatomic structures, and organs at risk. Int J Radiat Oncol Biol Phys 2006; 64: 1380-1388.

9. Schmid MP, Fidarova E, Pötter R et al. Magnetic resonance imaging for assessment of parametrial tumour spread and regression patterns in adaptive cervix cancer radiotherapy. Acta Oncol 2013; 52: 1384-1390.

10. Haie-Meder C, Pötter R, Van Limbergen E et al. Recommendations from Gynaecological (GYN) GEC-ESTRO Working Group (I): concepts and terms in 3D image based 3D treatment planning in cervix cancer brachytherapy with emphasis on MRI assessment of GTV and CTV. Radiother Oncol 2005; 74: 235-245.

11. Pötter R, Haie-Meder C, Limbergen EV et al. Recommendations from gynaecological (GYN) GEC ESTRO working group (II): Concepts and terms in 3D image-based treatment planning in cervix cancer brachytherapy-3D dose volume parameters and aspects of 3D image-based anatomy, radiation physics, radiobiology. Radiother Oncol 2006; 78: 67-77.

12. Lindegaard JC, Fokdal LU, Nielsen SK et al. MRI-guided adaptive radiotherapy in locally advanced cervical cancer from a Nordic perspective. Acta Oncol 2013; 52: 1510-1519.

13. Mazeron R, Fokdal LU, Kirchheiner K et al. Dose-volume effect relationships for late rectal morbidity in patients treated with chemoradiation and MRI-guided adaptive brachytherapy for locally advanced cervical cancer: Results from the prospective multicenter EMBRACE study. Radiother Oncol 2016; 120: 412-419.

14. Tanderup K, Eifel PJ, Yashar CM et al. Curative radiation therapy for locally advanced cervical cancer: brachytherapy is NOT optional. Int J Radiat Oncol Biol Phys 2014; 88: 537-539.

15. Georg P, Pötter R, Georg D et al. Dose effect relationship for late side effects of the rectum and urinary bladder in magnetic resonance image-guided adaptive cervix cancer brachytherapy. Int J Radiat Oncol Biol Phys 2012; 82: 653-657.

16. Fokdal L, Sturdza A, Mazeron R et al. Image guided adaptive brachytherapy with combined intracavitary and interstitial technique improves the therapeutic ratio in locally advanced cervical cancer: Analysis from the retroEMBRACE study. $R a-$ diother Oncol 2016; 120: 434-440. 